\title{
Testicular aspiration of sperm for intracytoplasmic sperm injection: an alternative treatment to electro-emission: case report
}

\author{
W Watkins, T Lim, H Bourne, HWG Baker and B Wutthiphan \\ Royal Womens Hospital, Melbourne, Australia and Austin and Repatriation Medical Centre, Melbourne, Australia
}

The quantity and quality of spermatozoa produced by electro- or vibro-ejaculation in spinal cord injury patients is often poor, thus advanced reproductive techniques are required if pregnancy is to be achieved. On other occasions no spermatozoa are obtained. We present a successful case of pregnancy achieved using spermatozoa aspirated directly from the testicle combined with intracytoplasmic sperm injection in an in vitro fertilization cycle. We propose this as an alternative to electroejaculation in selected patients.

Keywords: testicular sperm; spinal cord injury; electro-ejaculation; pregnancy

\section{Introduction}

Spontaneous ejaculation is uncommon following spinal cord injury (SCI) in all but those with very incomplete lesions. While reflex erections are common, it is estimated that less than $5 \%$ of SCI male patients are able to reproduce unaided by medical intervention. ${ }^{1}$

Eighty percent of male SCI patients admitted to the Austin Spinal Unit are aged between 16 and 35 years. Semen quality may deteriorate as early as 2 weeks after SCI. ${ }^{2}$ As a result, one of the major disabilities that results from SCI is the loss of fertility. The Spinal Injury Clinic of the Austin and Repatriation Medical Centre offer semen collection after acute spinal cord injury to all male patients between the ages of 16 and 40 years. This has only been clinic practice since 1993, and is not always possible due to the patients' medical status. There are therefore a large number of patients with chronic SCI that have not been offered the opportunity of acute phase semen collection. The vast majority of patients will require assistance by means of vibro- or electro-ejaculation for semen collection. In these situations, the quality of semen produced is often poor with small volumes and variable sperm concentrations, and in particular poor sperm motility and viability.

Various protocols have been established for the implementation of assisted ejaculation in SCI patients. We favour a protocol of consecutive day ejaculation for at least 3 days, or until suitable quality sperm has been obtained. The semen may be used fresh or cryopreserved for later use by means of artificial insemination or other means of assisted reproduction.

Correspondence: William B Watkins, FRACOG, Reproductive Biology Unit, Royal Womens Hospital, 132 Grattan St., Carlton, 3055, Victoria, Australia
Where vibro-ejaculation has failed, electro-ejaculation using a rectal probe, with or without general anaesthesia, is used.

Assisted ejaculation fails in some patients, particularly in those with lower motor neuron lesions. ${ }^{3}$ In other patients extremely poor semen profiles are obtained, suitable only for assisted reproductive procedures. The advent of intracytoplasmic sperm injection (ICSI) which is rapidly becoming available in most IVF units, has meant that even in the most severe cases, successful fertilisation and reproduction may occur.

We present an alternative to electro-ejaculation, where such procedures had resulted in extremely poor quality semen.

\section{Case report}

A 26 year old patient and his 26 year old wife presented for assistance with future fertility. The patient suffered a diving accident 3 years previously resulting in tetraplegia which was motor incomplete below $\mathrm{C}_{5}$, and motor complete below $\mathrm{C}_{7}$. At the time of the accident his wife was 7 months pregnant with their first child. The first pregnancy was completed successfully and the couple were interested in adding to their family.

Examination revealed testes at the lower limit of normal size: $15 \mathrm{mls}$ each. Serum FSH was normal. Vibro-ejaculation failed to result in a specimen being produced and due to the preservation of visceral sensation the patient was admitted for general anaesthetic and rectal electro-ejaculation on three successive days.

On each occasion extremely poor samples were obtained. Volumes produced were $<1 \mathrm{ml}$ on each occasion and sperm concentrations $<1 \times 10^{6} / \mathrm{ml}$. A 
high level of debris was present with only occasional, poorly motile sperm seen per high power field. No improvement was evident between the first and third samples and while they were deemed suitable for microinjection it was felt that freezing of such a sample would be unsuccessful.

The patient was informed of these findings and given the option of further electro-ejaculation or retrieval of sperm by testicular biopsy, appropriately timed with an oocyte collection procedure. The patient elected to undergo testicular biopsy.

Ovarian stimulation was obtained using leuprolide acetate (Lucrin; Abbott, Kurnell, NSW, Australia) daily for luteal phase down regulation. Once down regulation was obtained Metrodin 150 IU (Serono, French's Forest, NSW, Australia) was added to achieve ovarian stimulation. Human chorionic gonadotropin 5000 IU (Serono, Australia) was given $37 \mathrm{~h}$ prior to transvaginal oocyte retrieval.

Testicular aspiration was performed following oocyte retrieval. The procedure and subsequent method of processing the sample has been described in detail elsewhere. ${ }^{4,5}$ Briefly, the skin of the scrotum was prepared with $0.1 \%$ chlorhexidine and testicular anaesthesia obtained by injecting $5 \mathrm{ml}$ lignocaine $1 \%$ into the spermatic cord, around the vas, approximately $4-6 \mathrm{~cm}$ above the testicle. Adequate anaesthesia was obtained in approximately $10 \mathrm{~min}$. The testicle was held firmly in one hand and a 20 gauge $5 \mathrm{~cm}$ Menghini needle with stylet introduced into the testicle to a depth of approximately $0.5 \mathrm{~cm}$, taking care to avoid the epididymis. A $20 \mathrm{ml}$ plastic syringe with the plunger withdrawn to the $5 \mathrm{ml}$ mark was attached and further withdrawn to the $10 \mathrm{ml}$ mark to obtain negative pressure. The needle and syringe were then advanced and withdrawn through the testicle three times using short thrusting movements in order to cut the testicular tissue. The needle and syringe were then withdrawn from the testicle maintaining negative pressure in the syringe. The needle and syringe was then flushed with HEPES-buffered human tubal fluid (HTF; Irvine Scientific, Irvine CA). Several sections of seminiferous tubule were obtained.

The specimen was then transferred to a small petri dish (Falcon 3001; Becton Dickinson, Lincoln Park, NJ, USA) containing HEPES/HTF supplemented with $4 \mathrm{mg} / \mathrm{ml}$ human serum albumin (Australian Red Cross) and dissected using fine scissors and 25-gauge needles. The tissue pieces were then pipetted vigorously to disperse the sperm and the resulting suspension transferred to a conical test-tube (Falcon 2099; Beckton Dickinson). The suspension was then allowed to stand briefly, for less than $5 \mathrm{~min}$, to allow the large tissue clumps to settle to the bottom of the tube. The top layer of the suspension was then transferred to a fresh tube and centrifuged at $1800 \mathrm{~g}$ for $5 \mathrm{~min}$ and the resulting pellet resuspended in a small volume $(0.5 \mathrm{ml})$ of albumin supplemented HEPES/HTF, prior to being examined.

For use, approximately $0.3 \mathrm{ml}$ of the final sperm suspension was spread onto a plastic petri dish (Falcon 1006, Becton Dickson) along with an additional small drop of fresh albumin supplemented HEPES/HTF. The dish was then covered with $6 \mathrm{ml}$ of temperature equilibrated mineral oil (Sigma, St. Louis, USA) and left at $37^{\circ} \mathrm{C}$ until needed for injection. At this point the majority of spermatozoa were immotile, but sufficient numbers became motile with further culture. The micro injection procedure has been previously described. ${ }^{5}$

\section{Results}

Six mature oocytes were obtained and ICSI was performed using sperm obtained by testicular biopsy. Three oocytes fertilized normally and cleaved on day 2 . Two embryos were transferred to the uterine cavity on day 2 and one was frozen. Serum beta-HCG 17 days post embryo transfer was positive and transvaginal ultrasound at 6 weeks gestation revealed a single foetal heart. No complications were encountered with either procedure.

\section{Discussion}

In the majority of cases, vibro-ejaculation will be successful in obtaining sperm for use in a variety of fertility treatments. Where this fails, electroejaculation will usually enable an ejaculate to be collected. At times however, spermatozoa may not be obtained for a variety of reasons, including failure to elicit ejaculation, unexpected genital tract obstruction or a spermatogenic disorder. In addition, the procedure requires general anaesthesia where visceral sensation is present, multiple staff members and entails the small risk of rectal perforation or mucosal burns.

In the case presented, local anaesthesia was used due to the presence of sensation. Due to the presence of autonomic dysreflexia we would recommend the use of local anaesthesia even where the sensory lesion is complete.

We have used testicular biopsy to obtain histological specimens of testicular tissue on over 300 occasions without significant side effects, and have had a high level of patient acceptance. More recently we have used the same procedure to obtain spermatozoa in cases of obstructive azoospermia for use in our microinjection assisted reproduction programme. ${ }^{5}$

Various explanations have been proposed for the observation of poor semen profiles in men with SCI. Adverse factors may include increased scrotal temperature, urinary tract infection, functional obstruction or stagnation, neural effects on testicular and epididymal physiology, sperm autoimmunity, and external trauma/pressure effects. Reactive oxygen species (ROS) have recently been shown to be present in the semen of $97 \%$ of patients with SCI, $90 \%$ of which continued to produce reactive oxygen species after Percoll washing. The presence of ROS has been associated with reduced sperm motility, abnormal 
sperm morphology, and decreased sperm-egg interaction. ${ }^{6}$ However, there is as yet insufficient data to determine whether the presence of high levels of ROS in these patients will have a detrimental effect on the fertilization or implantation rate in such patients treated with ICSI. It is possible that sperm extracted from the testicle would be free from the detrimental effects of ROS.

The introduction of ICSI has significantly improved the prospects in reproduction for SCI patients with poor semen profiles. The semen profile is similar to that seen in the condition of necrospermia, a rare cause of infertility in men without SCI. The sperm characteristically has motility $<20 \%$ with commonly $<5 \%$ motile sperm. The percentage of live sperm as determined by exclusion of eosin-Y is $<30 \%$. Electronmicroscopy reveals severe degenerative changes in ejaculated spermatozoa but normal ultrastructure of late spermatids in the testis.

Aspiration from the vas deferens has also been reported as a successful method of obtaining spermatozoa from SCI patients for use in assisted reproduction. ${ }^{7}$ We have found testicular biopsy to be a very satisfactory means of obtaining spermatozoa for assisted reproduction though others have found a reduced fertilization rate using testicular sperm. ${ }^{8}$ Testicular biopsy has the advantage over other surgical means of sperm retrieval ie, micro-epididymal sperm aspiration (MESA) in that it is a simple technique requiring a minimum of surgical skill, the procedure may be performed in the office setting, and requires only local anaesthesia. On all occasions to date we have obtained adequate numbers of spermatozoa for injection, even where a previous biopsy had shown severe hypospermatogenesis. Furthermore, on occasions where spermatogenesis had been so poor that no live spermatozoa have been present in an ejaculated specimen, we have been able to obtain motile sperm directly from the testis.

In conclusion, testicular biopsy provides an alternative means of sperm retrieval in cases of SCI where assisted ejaculation has been unsuccessful in producing semen samples suitable for alternative methods of assisted reproduction, or where electro-ejaculation is unavailable.

\section{References}

1 Talbot HS. The sexual in paraplegia. J Urol 1955; 73: 91.

2 Mallidis C et al. Collection of semen from men in acute phase spinal cord injury. Lancet 1994; 343: 1072-1073.

3 Buch JP. Evaluation and treatment of infertility in spinal cord injured men through rectal probe electroejaculation. J Urol 1993; 149: $1350-1354$

4 Mallidis C, Baker HWG. Fine needle tissue aspiration biopsy of the testis. Fertil Steril 1994; 61: 367-374.

5 Bourne H, Watkins W, Speirs A, Baker H. Pregnancies after intracytoplasmic injection of sperm collected by fine needle biopsy of the testis. Fertil Steril (in press).

6 Lamirande E. Increased reactive oxygen species formation in semen of patients with spinal cord injury. Fertil Steril 1995; 63: $637-642$.

7 Burch JP. Greatly improved sperm motility from vas deferens sperm retrieval: a case of accessory gland related subfertility in spinal cord injured man. A case report. Paraplegia 1994; 32: $501-504$.

8 Nagy $\mathrm{Z}$ et al. Using ejaculated, fresh, and frozen-thawed epididymal and testicular spermatozoa gives rise to comparable results after intracytoplasmic sperm injection. Fertil Steril 1995; 63: $808-815$. 